\title{
Right Ear
}

National Cancer Institute

\section{Source}

National Cancer Institute. Right Ear. NCI Thesaurus. Code C81285.

The organ of hearing located on the right side of the head. 